\title{
Chaotic Universe dynamics using a Fokker-Planck equation
}

\author{
David H. Coule \\ and \\ Kirk O. Olynyk \\ Fermi National Accelerator Laboratory \\ P.O. Box 500, Batavia, Il 60510
}

\begin{abstract}
A Fokker-Planck equation that accounts for fluctuations in field and its conjugate momentum is solved numerically for the case of a $\lambda \phi^{4}$ potential. Although the amount of inflation agrees closely with that expected classically, in certain cases (large initial fields or large dispersions) the "slow rolling" approximation appears invalid. In such cases inflation would stop prematurely before possibly restarting.
\end{abstract}


The new inflationary universe model [1] is able to solve a number of important cosmological problems. It has been argued that in order to account for the observed fluctuations in the matter density spectrum, the field causing infation has to be very weakly coupled [2]. This results in high temperature effects being irrelevant for the behaviour of the field. It is therefore surprising that the field should be sitting in just the right place when spontaneous symmetry breaking occurs to produce a false vacuum state.

However Linde's chaotic universe model [3] suggests that phase transitions are not necessary for inflation: all what is needed is for the initial field to be displaced sufficiently from its minimum. In order for chaotic inflation to occur the scalar potential $V(\phi)$ must be larger than i) kinetic energy $\left(\partial_{o} \phi\right)^{2}$ and ii) spatial variations $\left(\partial_{i} \phi\right)^{2}$. In fact the first condition is not serious since if the kinetic energy is large it redshifts rapidly with time $t$ : as $\sim 1 / t^{2}$, whereas the field decreases only as $\log (t)$ enabling the potential to eventually dominate [4]. Therefore we only need to consider a domain (of size $\Delta l \sim M_{p}^{-1}$ ) with $\left(\partial_{i} \phi\right)^{2}<V(\phi)$; see however [5]. It should also be mentioned that inflation can also result from higher order corrections to Einstein's field equation ( $R^{2}$ or conformal anomaly terms) provided the initial value of the Ricci scalar is large enough [6].

In the context of the new inflationary universe model Vilenkin [7] argued that a distribution function $W$ for the scalar field is an important quantity 
for characterizing the dynamics. $W$ satisfies a diffusion equation while in the false vacuum state: the coefficient of diffusion $D$ is related to the Hubble parameter $\left(D=H^{3} / 8 \pi^{2}\right)$. When the slope of the potential is included a Fokker-Planck equation results [8-13]

$$
\frac{\partial W}{\partial t}=\frac{\partial}{\partial \phi}\left(\frac{V^{\prime}}{3 H} W\right)+\frac{\partial^{2}}{\partial \phi^{2}}\left(\frac{H^{3}}{8 \pi^{2}} W\right), \text { where } V^{\prime}=\frac{\partial V}{\partial \phi}
$$

Graziani and Olynyk [9] found that for a "Mexican hat" Higgs potential fluctuations in momentum could dramatically affect the evolution of the field during new inflation. Following their approach, we develop the FokkerPlanck equation for a $\lambda \phi^{4}$ potential while improving upon their work by making the Hubble term a function of the scalar field.

We assume the distribution function is Gaussian to simplify computations. The inclusion of an initial dispersion $\sigma$ is expected to increase the mean velocity since the classical force $\partial V / \partial \phi$ is now greater. For a $\lambda \phi^{4}$ potential any initial dispersion will tend to decrease as the field rolls down the potential, but if fluctuations are large enough the dispersion can increase instead. In fact, a small increase in dispersion can result in an eternal universe since the number of "trials" is so large $\sim e^{3 H t}$ that some domains have large fields and are always inflating [7,14-17].

Previous work on solving eq.(1) numerically for chaotic inflation has been carried out by Bardeen and Bublick for the case of a massive scalar field [12]: they found a broadening of the distribution, which they mention can have an effect on the roll down time. 
The Hubble constant is determined from one of the Einstein equations, and ignoring the curvature term is given by $[1,3]$

$$
H^{2}=\frac{8 \pi}{3 M_{p}^{2}}\left(\frac{p^{2}}{2}+V(\phi)\right)
$$

where $p=\dot{\phi}$. If we assume that the kinetic energy is much smaller than the potential energy term, then we may make the following approximation for the Hubble term:

$$
H^{2} \approx \frac{\phi^{4}}{h^{2}}, \text { where } h^{2}=\frac{3 M_{p}^{2}}{2 \pi \lambda}
$$

We always check that the solution of the equations of motion are consistent with the approximation of small conjugate momentum of the scalar field.

It has been demonstrated by Wigner that quantum mechanics can be formulated in terms of a Fokker-Planck type equation. Although this representation is not well known, it is completely equivalent to the familiar Heisenberg or Schrodinger pictures of quantum mechanics. To lowest order in $\hbar$, the Fokker-Planck equation can be recast as a stochastic differential equation, a Langevin equation. We shall present the derivation of the equations of motion for this semiclassical (order $\hbar$ ) case. However, it should be remembered that the result is a semiclassical approximation to a fully correct formulation of quantum mechanics.

The Langevin equation corresponding to the Linde-Albrecht-Steinhardt equation [1] for the long wavelength modes of the scalar field is ${ }^{1}$

\footnotetext{
${ }^{1}$ neglecting spatial gradients, for a discussion of this see [12].
} 


$$
\ddot{\phi}=-3 H \dot{\phi}-\frac{d V(\phi)}{d \phi}+\eta(t)
$$

The noise term $\eta(t)$ is assumed to have a Gaussian nature, that is, the ensemble averages of the noise terms must satisfy

$$
\begin{aligned}
\langle\eta(t)\rangle & =0 \\
\left\langle\eta\left(t_{1}\right) \eta\left(t_{2}\right)\right\rangle & =N \delta\left(t_{1}-t_{2}\right)
\end{aligned}
$$

To give $N$ a value, we have applied this stochastic analysis to the motion of a free massless scalar field in de Sitter space. One finds that initially $\left\langle\phi^{2}\right\rangle \simeq N t /(3 H)^{2}$. In order for this result to agree with the solution that uses the quantum field theory methods [18] we require

$$
N=\frac{9 H^{5}}{4 \pi^{2}}
$$

The Fokker-Planck corresponding to the Langevin eq.(4) has the general form

$$
\frac{\partial W}{\partial t}=-\frac{\partial(p W)}{\partial \phi}-\frac{\partial(\dot{p} W)}{\partial p}+\frac{1}{2} \frac{\partial^{2}(N W)}{\partial p^{2}}
$$

To simplify this equation we use the approximation for the Hubble term, so that eq.(4) can be written as

$$
\dot{p}=-3 \frac{\phi^{2} p}{h}-\lambda \phi^{3}+\eta(t)
$$

Rescaling the variables such that

$$
\begin{aligned}
t & \equiv b^{-1} \bar{t} \\
\phi & \equiv a \bar{\phi}
\end{aligned}
$$


Leads to the dimensionless equation

$$
\frac{d \bar{p}}{d \bar{t}}=-\frac{3 a^{2}}{b h} \bar{\phi}^{2} \bar{p}-\frac{\lambda a^{2}}{b^{2}} \bar{\phi}^{3}+\bar{\eta}(\bar{t})
$$

where $\bar{\eta}(\bar{t}) \equiv a^{-1} b^{-2} \eta(t)$. We are free to choose the scales $a$ and $b$ as we wish. A particularly convenient choice is defined by $3 a^{2}=b h$ and $\lambda a^{2}=b^{2}$. This leads to a simple Langevin equation given by

$$
\frac{d \bar{p}}{d \bar{t}}=-\bar{\phi}^{2} \bar{p}-\bar{\phi}^{3}+\bar{\eta}(\bar{t})
$$

The order $\hbar$ Fokker-Planck equation corresponding to this simplified dimensionless Langevin equation is -cf. eq.(1)

$$
\begin{aligned}
\frac{\partial W}{\partial t}= & -\frac{\partial(p W)}{\partial \phi}+\frac{\partial}{\partial p}\left[\left(\phi^{2} p+\phi^{3}\right) W\right] \\
& +\left(\frac{\epsilon}{2}\right) \frac{\partial^{2}}{\partial p^{2}}\left(\phi^{10} W\right)
\end{aligned}
$$

where $\epsilon=\lambda / 108 \pi^{2}$. The bars over the dimensionless $\phi$ 's and $p$ 's have been neglected for notational clarity. From this point one should assume that the fields are dimensionless unless indicated otherwise.

From eq.(12) we can make some general remarks as to when we expect fluctuations induced by the diffusion term to become important. Assuming $\phi^{2} p \sim \phi^{3}$ in the 2 nd term on the RHS which we compare with the final term i.e.

$$
\phi^{3} \sim \lambda \frac{\phi^{10}}{108 \pi^{2} p} \sim \frac{\lambda \phi^{9}}{108 \pi^{2}}
$$


so that we expect fluctuations to be important when $\phi>\lambda^{-1 / 6}$.

Another scale when fluctuations become important can be seen from eq.(6) when the diffusion term is large, that is when $H>1$ which occurs when $\lambda \phi^{4}>1$ i.e. $\phi>\lambda^{-1 / 4}$, however this size field corresponds to Planck energy densities.

By using a general equation of motion for the moments of the variables $p$ and $\phi$ we can convert eq.(12) into a set of coupled ordinary differential equations. This is not an approximation, however, this means that an infinite set of coupled ordinary differential equations is generated, coupling an infinite tower of modes. We therefore make the standard approximation that not all the modes are independent by assuming that the scalar field distribution in the $\phi$-direction is Gaussian. All of the Gaussian approximations may be summarized as the expression of the expectation values $\left\langle\phi^{n}\right\rangle$ as functions of $\langle\phi\rangle$ and $\sigma \equiv\left\langle(\phi-\langle\phi\rangle)^{2}\right\rangle$. A set of five coupled non-linear ordinary differential equations in terms of the variables $\left[\langle\phi\rangle,\langle p\rangle,\left\langle\phi^{2}\right\rangle,\left\langle p^{2}\right\rangle,\langle\phi p\rangle\right]$ are obtained. The equations of motion are straight forward to derive and the numerical solutions were obtained using standard algorithms.

We present here the solution for the case of $\lambda=10^{-4}$. We have confirmed that similar behavior is found for $\lambda=10^{-8}$ and $10^{-12}$ with the scalar field appropriately scaled. The dispersion $\sigma$ of the distribution is plotted against the mean field $\langle\phi\rangle$ for various initial field values in Fig.(1). The linewidth of the Gaussian distribution $\sim \sigma^{1 / 2}$ first increases as the mean 
field moves towards the origin; it then decreases due to the classical tendency to contract once the quantum fluctuations become less significant. Usually, the dispersion in momentum decreases as the distribution moves towards the origin. However, for large initial fields $\langle\phi\rangle$, the momentum fluctuations are found to grow until the lines are discontinued when $p^{2} \sim \lambda \phi^{4}$ (in our units when $\left\langle p^{2}\right\rangle \sim\left\langle\phi^{4}\right\rangle$ ). At such time the approximation for the Hubble parameter in eq.(2) is no longer valid and the de Sitter stage ends.

The Planck energy density $\sim M_{p}^{4}$ occurs in our units for $\langle\phi\rangle \sim 40$ (since the scaling constant $a$ is $\sim 0.25$ ). For such $\phi$ the dispersion is seen to rapidly increase: it has been suggested that this might be interpreted as creation of an inflationary universe by diffusion from space-time foam $[16,17,8]$.

In Fig.(2) the initial field $\langle\phi\rangle$ is fixed at 5 and its evolution is followed for various initial dispersions. The dispersion decreases as the distribution evolves for small initial $\sigma$ 's but, as the dispersion is increased the "diffusion term" takes over and the dispersion increases instead. Again the momentum fluctuations stop the de Sitter stage, indicated by the discontinued lines.

According to the results of [4] once inflation has finished due to large momenta the kinetic energy could redshift away rapidly while the field decreases only slightly so that once again $\lambda \phi^{4} \gtrsim p^{2}$, and the de Sitter stage would resume. A possible trajectory is represented by the dotted line in 
Fig.(1) during which the kinetic energy decays before the de Sitter stage restarts at $A$. This might proceed a number of times but so long as the total expansion during the de Sitter stages is $Z 70$ e-foldings the flatness and horizon problems would still be solved.

Unlike for the Mexican hat potential where the fluctuations in momentum could dramatically slow down the evolution of the field [9] no such effect occurs for this simple $\lambda \phi^{4}$ potential. In fact the field evolves slightly faster than the pure classical motion would suggest but the effect is very small $\leqslant 5 \%$, which is in agreement with the results of [12]. However, we have found that for large or broad initial fields the "slow rolling" approximation is inadequate. Certain "creation of the universe" solutions to eq.(1) $[14,16,17,13,8]$ may need to be modified.

Ideally we would like to solve eq.(7) with an exact Hubble parameter which included both kinetic and potential terms. Alternatively, the Langevin equation (eq.(4)) might be solved directly using a Monte Carlo method. The problem is further compounded by the fact that the underlying metric need not be constant: a time dependent noise term is required.

This work was supported in part by the DOE and NASA through Fermilab. 


\section{References}

1. A.D. Linde, Phys. Lett. 108B (1982) 389; $114 \mathrm{~B}$ (1982) 431; 116B (1982) 335;

A. Albrecht and P.J. Steinhardt, Phys. Rev. Lett. 48 (1982) 1220

2. V.F. Mukhanov and G.V. Chibisov, JEPT Lett. 33 (1981) 532;

A.A. Starobinsky, Phys. Lett. 117B (1982) 175;

A.H. Guth and S.Y. Pi , Phys. Rev. Lett. 49 (1982) 1110;

J. Bardeen, P.J. Steinhardt and M.S. Turner, Phys. Rev. D28 (1983) 679 ;

S.W. Hawking, Phys. Lett. 115B (1982) 295

3. A.D. Linde, Phys. Lett. 129B (1983) 177 ; see also the reviews:

A.D. Linde, Rep. Prog. Phys. 47 (1984) 925;

R.H. Brandenberger, Rev. Mod. Phys. 57 (1985) 1;

M.S. Turner, The inflationary paradigm, Fermilab preprint (1986);

R.H. Brandenberger, J. Mod. Phys. 2A (1987) 77

4. A.D. Linde, Phys. Lett. 162B (1985) 281;

T. Piran, On general conditions for inflation, preprint (1985);

B.C. Paul, D.P. Datta and S. Mukherjee, Chaotic universe scenario in an anisotropic universe, North Bengal University preprint (1986) 
5. A.S. Goncharov, Phys. Lett. 165B (1985) 49;

L.A. Khalfin, Sov. Phys. JEPT 64 (1986) 673

6. A.A. Starobinsky, Phys. Lett. 91B (1980) 99;

A.A. Starobinsky, Sov. Astrom. Lett. 9 (1983) 302;

A. Vilenkin, Phys. Rev. D32 (1985) 2511;

M. Mijic, M. Morris and W. Suen, Phys. Rev. D34 (1986) 2934;

L.A. Kofman, A.D. Linde and A.A. Starobinsky, Phys. Lett. 157B (1985) 361

7. A. Vilenkin, Phys. Rev. D27 (1983) 2848

8. A.A. Starobinsky, in: Field Theory, Quantum gravity and Strings, eds. H.J. de Vega and N. Sanchez (Springer-Verlag, Heidelberg, 1986) p.107

9. F.R. Graziani and K.O. Olynyk, Fermilab preprint 85/175-T (1985); revision in preparation (1987)

10. A.S. Goncharov and A.D. Linde, Sov. J. Part. Nucl. 17 (1986) 837

11. S. Rey, Caltech preprint $68-1371$ (1986)

12. J.M. Bardeen and G.J. Bublick, Seattle preprint 40048-OI C6 (1986)

13. M.D. Pollock, Trieste preprint IC/86/69 (1986) 
14. A.D. Linde, Phys. Lett. 175B (1986) 395

15. A.D. Linde, Mod. Phys. Lett. A 1 (1986) 81

16. A.D. Linde, in: 300 Years of Gravitation, eds S.W. Hawking and W. Israel (Cambridge University Press, Cambridge, 1987);

A.D. Linde, in: Proceedings of the Noble Symposium on Elementary Particles, Physica Scripta 34 (1986)

17. A.S. Goncharov, A.D. Linde and V.F. Mukhanov, The global structure of the inflationary universe, Lebedev Institute preprint (1987)

18. A. Vilenkin and L. Ford, Phys. Rev. D26 (1982) 1231;

A.A. Starobinsky, Phys. Lett. 117B (1982) 175;

A.D. Linde, Phys. Lett. 116B (1982) 335;

A. Vilenkin, Nucl. Phys. B226 (1983) 527;

S.W. Hawking, Phys. Lett. 115B (1982) 


\section{Figure Captions}

Fig. 1) The evolution of the dispersion $\sigma$ as a function of mean field $\langle\phi\rangle$. The lines stop when $\left\langle p^{2}\right\rangle \sim\left\langle\phi^{4}\right\rangle$ which indicates that the approximation for the Hubble parameter is no longer valid. The dotted line indicates a possible path during which the kinetic energy decreases before resuming a de Sitter expansion at A. As the Planck energy density is reached $\langle\phi\rangle \sim 40$ the dispersion starts to increase dramatically: rapid diffusion regime.

Fig. 2) The evolution of the distribution for fixed initial field $\langle\phi\rangle=5$ and various initial dispersions. The trajectories are again discontinued once $\left\langle p^{2}\right\rangle \sim\left\langle\phi^{4}\right\rangle$. 


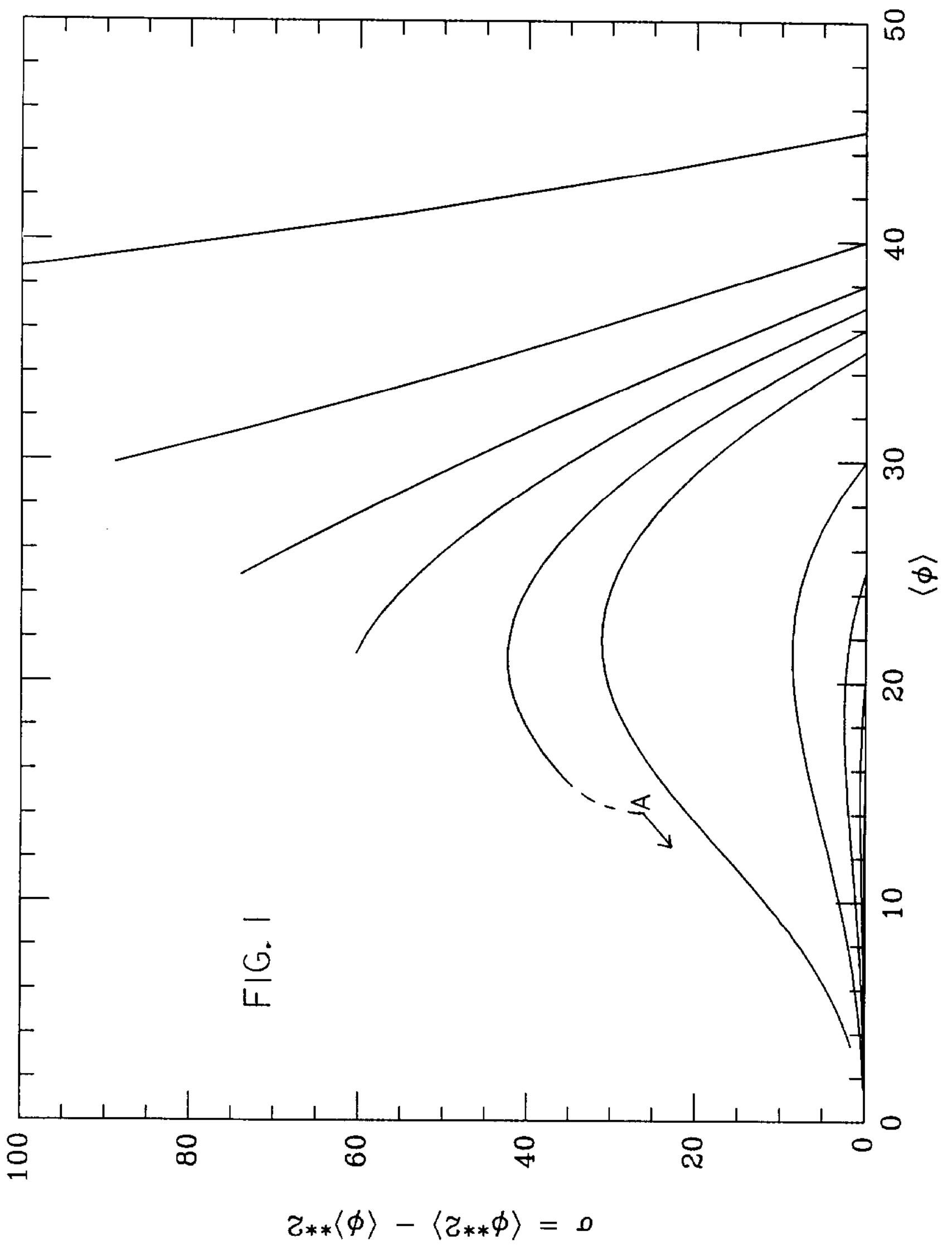




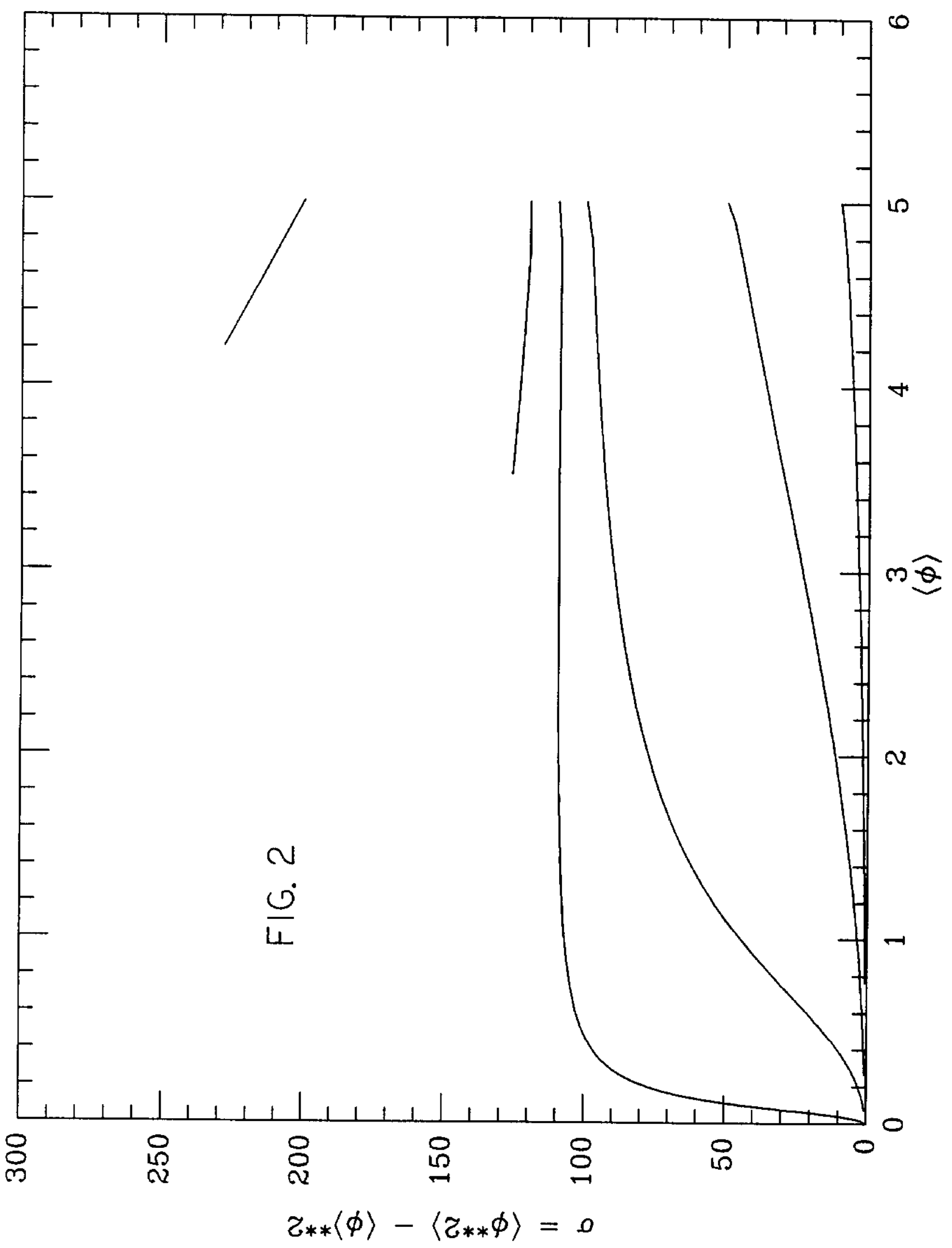

